\title{
GESTÃO PARTICIPATIVA E A INTERFACE COM TRABALHADORES DO SISTEMA ÚNICO DE SAÚDE: UMA REVISÃO INTEGRATIVA
}

\author{
J. B. L. CARVALHO, N. O. F. RAMOS, A. G. CÂMARA, G. A. TEIXEIRA, T. R. G. LOPES* \\ *Email: thaisrg12@hotmail.com \\ Universidade Federal do Rio Grande do Norte
}

Artigo submetido em outubro/2015 e aceito em junho/2016

DOI: $10.15628 /$ rbept.2016.3487

\begin{abstract}
RESUMO
Trata-se de uma revisão integrativa de literatura com objetivo de integrar o conhecimento produzido acerca da Gestão Participativa e a interface dos trabalhadores do SUS. Para seleção dos artigos foram consultadas três bases de dados - Scielo, LiLACS e MEDLINE - sendo incluídos doze artigos. Os resultados mostram estudos qualitativos, todos publicados entre 2008 a 2012. Identificou-se nos artigos que a Gestão Participativa é vivenciada por
\end{abstract}

trabalhadores de saúde, muito embora haja dificuldades para a mesma. Os resultados evidenciaram um maior número de estudos nas regiões sul e sudeste brasileiro. Conclui-se que a Gestão Participativa é importante para o desenvolvimento do SUS e que é possível superar desafios e fazer com que o trabalhador de saúde participe mais do processo de decisões.

PALAVRAS-CHAVE: Gestão Participativa; Trabalhadores; Sistema Único de Saúde.

\section{PARTICIPATIVE MANAGEMENT AND INTERFACE WITH EMPLOYEES OF THE UNIFIED HEALTH SYSTEM: AN INTEGRATIVE REVIEW}

\begin{abstract}
This is a literature integrative review in order to integrate the knowledge produced about the Participatory Management and the interface of SUS workers. To select the articles were consulted three databases - Scielo, LiLACS and MEDLINE being included twelve articles. The finds show qualitative studies, all published between 2008 and 2012. It was identified in articles that Participatory
\end{abstract}

Management is experienced by health workers, although there are difficulties for implant of that Management. Results revealed a greater number of studies in the regions south and southeast Brazil. It was concluded that Participatory Management is important for development of SUS and it is possible to overcome challenges and make the health worker participate more in decision process.

Keywords: Participatory Management; Workers; Single Health System 


\section{INTRODUÇÃO}

O Sistema Único de Saúde (SUS) começou seu processo de idealização a partir do movimento sanitarista no Brasil, no início da década de 1980, o qual foi marcado por discussões de uma parcela conscientizada da população formada, principalmente, por profissionais de saúde, que passaram a enxergar as diversas dimensões do processo de saúde/doença e que o Estado tem responsabilidade sobre a saúde do indivíduo e do coletivo. (BRASIL a, 2009)

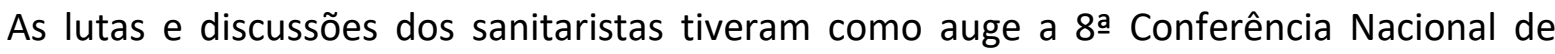
Saúde, realizada em 1986, a qual tinha como tema "saúde direito de todos e dever do estado". Na ocasião, os anseios da população quanto ao antigo sistema de saúde e os desejos por mudança foram expostos e evidenciou-se que o cuidado era uma responsabilidade também do governo e, sendo assim, todo e qualquer cidadão, independente de renda, deveria ter acesso a serviços de saúde de qualidade de forma gratuita. (BRASIL b, 2009)

Em 1988, foi promulgada a Constituição Federal do Brasil, a qual reafirma que a saúde é direito de todo e qualquer cidadão e um dever do estado, sendo essa conquista comemorada pela população. Somente após dois anos, as leis de no 8.080 e a 8.142 ambas de 1990, foram promulgadas sendo instituída a criação do SUS. (BRASIL b, 2009; BRASIL c, 2009)

Com essas leis foram instituída os princípios do SUS: universalidade, integralidade e equidade da assistência a saúde, nas quais asseguram a garantia da preservação da autonomia de cada indivíduo; o direito a informação; a divulgação no que se refere à abrangência do serviço de saúde para com o usuário; estabelecimentos das prioridades para alocação dos recursos; dispõem sobre a descentralização político-administrativa; a regionalização e a hierarquização da rede de serviços de saúde; além da participação da comunidade na gestão do sistema e no acompanhamento dos investimentos disponibilizados a ele. (BRASIL b, 2009; BRASIL c, 2009).

Desde sua idealização e sua concretização legislativa, na década de 1990, o SUS vem passando por constantes processos de aperfeiçoamento através da criação de aditivos em suas leis iniciais, projetos e estratégias. Reconhecendo a importância da gestão nesse processo de desenvolvimento do SUS foram criadas normas operacionais, a exemplo da NOB 93, que estimula a municipalização, voltada para a descentralização do poder monetário da escala federal para estadual e municipal, atribuindo mais responsabilidade e autonomia financeira aos gestores de cada instância. (CUNHA, 2012)

Com isso percebe-se que ao longo dos anos, o SUS apresentou avanços, aonde esses vêm contribuindo para sua melhor implantação no país. Seguindo com a certeza de que a descentralização do poder é o caminho para um sistema de saúde eficaz e almejando-se garantir que profissionais e usuários tivesse sua parcela de contribuição com o desenvolvimento do sistema de saúde do país, foi criada a Política Nacional de Gestão Estratégica e Participativa no SUS - ParticipaSUS, onde são descritas as funções dos gestores federais, estaduais e municipais e as responsabilidades atribuídas aos mesmos em integrar os profissionais nesse processo.(BEZERRA, 2012) 
A ParticipaSUS tem como princípios a valorização da participação social; a promoção da inclusão social de grupos específicos; a integração e ampliação dos serviços de auditoria e ouvidoria; o fortalecimento das formas coletivas de participação, sendo valorizada a participação dos usuários e dos trabalhadores. No que concerne a esse último grupo, a política valoriza a interlocução com os gestores, a participação em processos participativos de monitoramento e a participação dos mesmos na criação, gerenciamento, implementação e avaliação de estratégias que melhorem a participação popular e legitime os princípios do SUS.(BRASIL a, 2009)

Diante da importância da ParticipaSUS e a interface com os trabalhadores de saúde, surgiu o seguinte questionamento: Qual a produção de conhecimentos científicos acerca da Gestão Participativa e a interface com os trabalhadores do SUS? Com vista a contemplar tal questionamento, o objetivo do estudo foi integrar o conhecimento produzido acerca da Gestão Participativa e a inserção dos trabalhadores do SUS.

\section{MATERIAIS E MÉTODOS}

Para o alcance do objetivo, optou-se pelo método de revisão integrativa da literatura, uma vez que essa modalidade reúne e sintetiza o resultado de pesquisas de uma determinada temática, de maneira ordenada e sistematizada, contribuindo para o aprofundamento do tema em estudo. Seis etapas foram percorridas para a elaboração da presente revisão: elaboração da pergunta norteadora e objetivo do estudo; busca na literatura; avaliação dos dados; análise dos dados; discussão dos resultados, por fim, a apresentação dos resultados.

A busca foi realizada no mês de Novembro de 2013, nas bases de dados/bibliotecas virtuais: LILACS (Literatura Latino Americana e do Caribe em Ciências da Saúde), SciELO (ScientificElectronic Library OnLine) e MEDLINE. Cada base foi acessada por dois pesquisadores ao mesmo tempo, em diferentes computadores, a fim de garantir uma seleção melhor seleção e maior quantidade de artigos que respondessem ao questionamento de pesquisa.

Os descritores identificados nos Descritores em Ciências de Saúde (DesCS) foram trabalhadores e Sistema Único de Saúde; além deles foi utilizado o descritor aberto gestão participativa; os termos supracitados foram empregados na busca na literatura, nos seguintes cruzamentos: Gestão Participativa AND Trabalhadores AND Sistema Único de Saúde; Gestão Participativa AND Trabalhadores; Gestão Participativa AND Sistema Único de Saúde. Quanto ao recorte temporal, foram captadas todas as publicações disponíveis em cada base de dados até outubro de 2013, sem limite anterior, devido ao pequeno número de publicações que atendessem ao objetivo do estudo.

Os critérios de inclusão estabelecidos foram: artigos completos disponíveis eletronicamente de forma gratuita, publicados nos idiomas inglês, português, espanhol. Como critérios de exclusão: editoriais, notas ao editor, dissertações, teses, livros, opiniões de especialistas, estudos piloto e artigos que não respondessem ao questionamento proposto. Após a leitura criteriosa dos artigos encontrados e a aplicação dos critérios de inclusão e exclusão, a amostra constituiu-se de doze artigos lidos na íntegra (Tabela 1). 
Tabela 1 - Distribuição dos artigos encontrados e selecionados nas bases de dados eletrônicas. 2013.

\begin{tabular}{|c|c|c|c|}
\hline $\begin{array}{c}\text { Artigos/ } \\
\text { Base }\end{array}$ & Scielo & LiLacs & Medline \\
\hline Encontrados & 40 & 39 & 2 \\
\hline Excluídos & 30 & 37 & 2 \\
\hline Selecionados & 9 & 2 & 0 \\
\hline
\end{tabular}

Para caracterização dos dados, desenvolveu-se um formulário, o qual engloba referência do artigo, ano e local da publicação, delineamento do estudo e resultados encontrados. A discussão foi apresentada na forma descritiva, sendo os estudos analisados tendo como base a ParticipaSUS.

\section{RESULTADOS E DISCUSSÃO}

$\mathrm{Na}$ presente revisão integrativa, analisaram-se 11 artigos que atenderam aos critérios previamente estabelecidos. Todos os estudos foram realizados no Brasil, concentrando-se na região sudeste. As publicações ocorreram de 2004 a 2012, todos com delineamento metodológico descritivos. $O$ quadro 1 apresenta a caracterização desses estudos.

Quadro 1- Caracterização dos artigos segundo ano e local de publicação, resultados encontrados.

\begin{tabular}{|c|c|c|c|}
\hline Id. & Ano & Local da publicação & Resultados encontrados \\
\hline$A^{4}$ & 2012 & São Paulo/SP & $\begin{array}{l}\text { Reflexão acerca dos avanços da } \\
\text { Gestão Participativa e dos } \\
\text { avanços da participação dos } \\
\text { trabalhadores nos últimos } \\
\text { anos. }\end{array}$ \\
\hline$B^{6}$ & 2012 & São Paulo/SP & $\begin{array}{l}\text { Expõe expectativas e desafios } \\
\text { da Gestão Participativa e } \\
\text { mostra que é o "modelo } \\
\text { sustentável" que o SUS precisa. }\end{array}$ \\
\hline $\mathrm{C}^{7}$ & 2012 & Ribeirão Preto/SP & $\begin{array}{l}\text { Identifica dificuldades na } \\
\text { implantação de um modelo de } \\
\text { gestão participativa em um } \\
\text { hospital. }\end{array}$ \\
\hline$D^{8}$ & 2012 & São Paulo/SP & $\begin{array}{l}\text { Experiência da liga de ensino } \\
\text { de Medicina da Família na } \\
\text { gestão participativa. O artigo } \\
\text { enfoca a tríade ensino- } \\
\text { pesquisa-comunidade. }\end{array}$ \\
\hline $\mathrm{F}^{9}$ & 2011 & Rio de Janerio/RJ & $\begin{array}{l}\text { Expõe a importância do } \\
\text { trabalho em equipe e da } \\
\text { participação dos profissionais e } \\
\text { a estima pela mudança nos } \\
\text { serviços. }\end{array}$ \\
\hline & & & Demonstra a responsabilidade \\
\hline
\end{tabular}




\begin{tabular}{|c|c|c|l|}
\hline $\mathrm{G}^{10}$ & 2009 & Botucatu/SP & $\begin{array}{l}\text { dos trabalhadores na Gestão } \\
\text { Participativa e as dificuldades } \\
\text { encontradas no âmbito da ESF. }\end{array}$ \\
\hline $\mathrm{H}^{11}$ & 2009 & Porto Alegre/RS & $\begin{array}{l}\text { Reflexão acerca da participação } \\
\text { social e profissional em saúde. }\end{array}$ \\
\hline $\mathrm{I}^{12}$ & 2009 & $\begin{array}{l}\text { Reflexão acerca dos percalços } \\
\text { do Controle Social e da Gestão } \\
\text { Participativa. }\end{array}$ \\
\hline $\mathrm{J}^{13}$ & 2008 & Rio de Janeiro/RJ & $\begin{array}{l}\text { Relata a participação de } \\
\text { trabalhadores de saúde na } \\
\text { reorganização dos serviços. O } \\
\text { estudo aponta dificuldades e } \\
\text { como essas foram superadas. }\end{array}$ \\
\hline $\mathrm{K}^{14}$ & 2008 & Rio de Janeiro/RJ & $\begin{array}{l}\text { Apresenta reflexões acerca da } \\
\text { contribuição da educação } \\
\text { popular e saúde para a gestão } \\
\text { participativa do SUS. }\end{array}$ \\
\hline $\mathrm{L}^{15}$ & \multirow{2}{*}{2004} & São Paulo/SP & $\begin{array}{l}\text { Relata experiência de gerência } \\
\text { de enfermagem participativa e } \\
\text { oflexo positivo na qualidade } \\
\text { dos serviços. }\end{array}$ \\
\hline
\end{tabular}

A análise dos estudos possibilitou a identificação de fatores convergentes e divergentes na direção da gestão participativa, além de experiências exitosas de trabalhadores do SUS, apresentadas no quadro 2, em forma das categorias: avanços,dificuldades e desafios, e expectativas com as respectivas sub-categorias detalhando-as.

Quadro 2- Conhecimentos científicos acerca da Gestão Participativa e a interface com os trabalhadores do SUS, conforme as categorias.

\begin{tabular}{|c|c|}
\hline CATEGORIAS & SUBCATEGORIAS \\
\hline AVANÇOS $4,8,13,15$ & $\begin{array}{l}\text { - Fortalecimento do controle social, da gestão participativa e da valorização profissional no } \\
\text { âmbito do SUS; } \\
\text { - Ampliação do olhar crítico sobre a realidade social e do trabalho em saúde; } \\
\text { - Os trabalhadores do SUS referem com positividade este sistema, além de refletirem sua } \\
\text { prática constantemente, construindo coletivamente um novo olhar em relação ao SUS. } \\
\text { - A inserção da equipe de enfermagem na gerência dos serviços de Saúde pode propiciar o } \\
\text { desenvolvimento organizacional do sistema, inclusive aproximando o empregado do } \\
\text { empregador }\end{array}$ \\
\hline $\begin{array}{l}\text { DESAFIOS, } \\
\text { DIFICULDADES } \\
\text { 12-8, }\end{array}$ & $\begin{array}{l}\text { - A capacidade de lidar com o conflito nos espaços coletivos de gestão; } \\
\text { - Faltam ferramentas de gestão nas equipes e nos colegiados; } \\
\text { - A complexidade do cenário da saúde, pois no cotidiano dos serviços, o projeto de saúde } \\
\text { - está sempre em construção; } \\
\text { - A tomada de decisão compartilhada apenas parcialmente utilizada; }\end{array}$ \\
\hline
\end{tabular}




\begin{tabular}{|c|c|}
\hline & 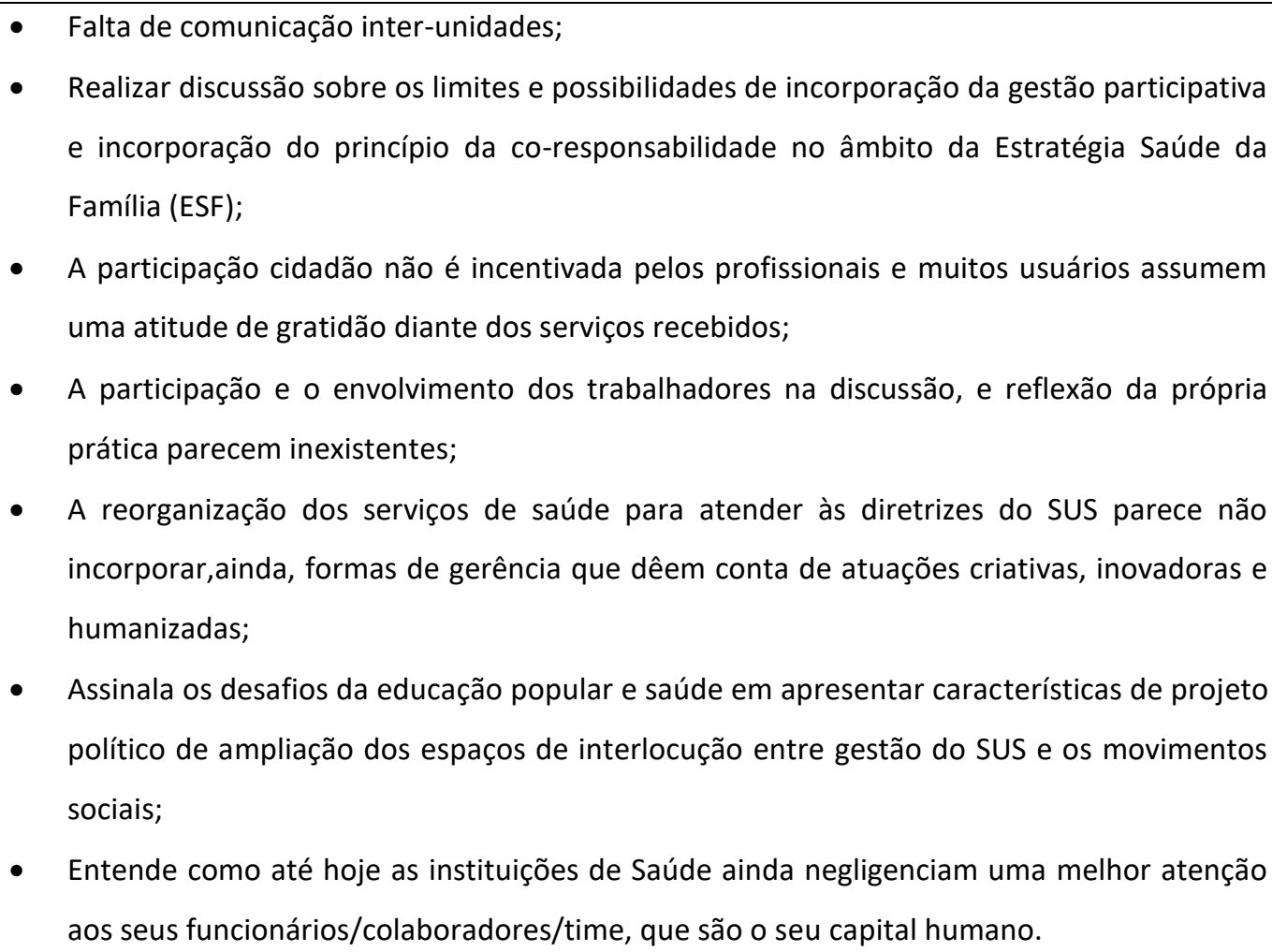 \\
\hline $\begin{array}{l}\text { EXPECTATIVAS } 6,8-9, \\
11-12,15\end{array}$ & $\begin{array}{l}\text { - Investir na construção de novos modos de produção da vida no plano coletivo, com } \\
\text { projetos baseados na igualdade e na convivência democrática; } \\
\text { - } \text { Encontrar uma forma efetiva de trabalhar em equipe que resulte numa atenção à saúde } \\
\text { integral e humanizada, porém prevalece uma postura de espera pelo sujeito das } \\
\text { mudanças; } \\
\text { - } \text { Encontrar uma forma efetiva de trabalhar em equipe que resulte numa atenção à saúde } \\
\text { integral e humanizada; } \\
\text { - } \text { Ampliar o grau de transversalização entre os sujeitos envolvidos no cuidado, numa } \\
\text { perspectiva de gestão em que todos sejam co-responsáveis pelos rumos da atenção } \\
\text { prestada; } \\
\text { - Suscitar debate e contribuir na elucidação das indagações sobre as possibilidades se } \\
\text { limites das instâncias de controle social do Sistema Único de Saúde; } \\
\text { A reflexão sobre as possibilidades de participação política no sus seja remetida ao } \\
\text { cotidiano institucional da gestão, pois o princípio de participação da comunidade diz } \\
\text { respeito à constituição do próprio direito à saúde; } \\
\text { Reflexão sobre as novas tendências gerenciais e como elas podem influenciar a } \\
\text { organização do trabalho de Enfermagem nos serviços de Saúde e as ações dos enfermeiros } \\
\text { gerentes em estratégias que atendam aos desafios da profissão, num contexto social em } \\
\text { transformação; }\end{array}$ \\
\hline
\end{tabular}




\section{de Saúde;}

- Incorporação de novos conhecimentos e habilidades, sintonizados a uma prática administrativa mais aberta, mais flexível e participativa, fundamentada não só na razão, mas também na sensibilidade e na intuição.

O ponto primordial para a mudança é assumir que a mesma é necessária. Em tese, o estado deu início a este processo. Através da criação das leis orgânicas, da realização das Conferências e Conselhos de Saúde, pela criação da NOB 93, reforçada pela NOB 96, a criação dos instrumentos de gestão como a Programação Anual de Saúde e o Relatório de Gestão e o próprio programa de humanização, entre outros programas e estratégias já lançadas pelas secretarias de Saúde o governo oferece as ferramentas para que essa a gestão participativa ocorra. (BRASIL a, 2009)

Porém, mesmo com a criação de leis e normas, que foram desenvolvidas com o objetivo de tornar a gestão mais dinâmica, onde haja interação e participação dos atores envolvidos, as unidades esbarram com um grande problema, a má administração. Ainda faltam profissionais capacitados para exercer essa função ou, muitas vezes, estes são substituídos por outros com determinadas tendências partidárias, similares a atual gestão estadual ou municipal. (GOULART, 2008)

Conforme identificado, é notório o desejo pela mudança por parte dos trabalhadores de saúde, muito embora uma boa parcela ainda não esteja disposta a se envolver para que aconteça. (GOULART, 2008)

Muitos deles estão profundamente habituados com o modelo de gestão tradicional e se sentem mais a vontade em simplesmente realizar as tarefas previamente determinadas, ou mecanizadas, a participar de seu desenvolvimento e construção.

Mesmo com todos os percalços enfrentados pelo sistema de saúde do país, podem-se citar diversas experiências exitosas encontradas nas publicações, ocorridas em diversos pontos do território nacional. Na cidade de Fortaleza-CE, duas universidades, uma pública e outra de âmbito particular, se uniram e criaram o Projeto de Extensão Liga da Saúde da Família. O projeto tem como iniciativa, integrar a educação em saúde, o serviço e a comunidade, potencializando assim os espaços de co-gestão docente assistencial. Nele, os idealizadores mostraram que o principal avanço resultante dessa estratégia foi o desenvolvimento e a ampliação do olhar crítico pelos integrantes da comunidade e, consequentemente, proporcionando satisfação à equipe criando o estímulo para continuar com o projeto. (BARRETO, 2012).

Quando se busca trabalhos científicos sobre a participação popular dentro da perspectiva de saúde coletiva, surgem estudos que afirmam a mudança ser mais rápida e eficaz quando cobrada e fiscalizada pela população, inclusive, várias implantações ocorreram em decorrência da participação popular.(BEZERRA, 2012; MAGALHÃES, 2004)

A importância dos conselhos locais de saúde, colegiados, as plenárias e fóruns de saúde, os quais começaram a ter contornos mais nítidos de órgãos fiscalizadores há apenas 10 anos, a partir da criação da Secretaria de Gestão do Trabalho e da Educação na Saúde (SGETES) e da Secretaria de Gestão Participativa - SGP (designação anterior da atual SGEP - Secretaria de Gestão Estratégica e Participativa) no ano de 2013. (PEDROSA, 2008) 
Cabe destacar que as publicações frisam que a SGETES investiu fortemente na educação popular e até março de 2014 pretende capacitar 31 mil conselheiros de saúde, além da finalização de cinco materiais de divulgação dos projetos da secretaria. Dentre as diversas atividades e funções da SGEP, é de competência da mesma articular as ações do Ministério da Saúde referentes à gestão estratégica e participativa, com os diversos setores governamentais e nãogovernamentais, relacionados com os condicionantes e determinantes da saúde. (PEDROSA, 2008)

Os desdobramentos destas competências estão diretamente ligados a implantação dos Sistemas Nacionais de Ouvidorias do SUS; de Monitoramento, Acompanhamento e Avaliação da Gestão do SUS e o de Auditoria do SUS. Todos eles refletem as condições do serviço ofertado à população e, consequentemente, estimulam a melhoria progressiva e superação das metas estipuladas pelo ministério da saúde. (PEDROSA, 2008)

As publicações apontam que é necessário distanciar o conceito de gerente, conhecido na administração, com o profissional gestor da área da saúde, para poder então obter um melhor desempenho das atividades da equipe e da unidade como um todo. Espera-se que, o quanto antes, as instituições se afastem dos modelos de gerenciamento tradicionais e proporcionem aos funcionários maior autonomia e multidisciplinaridade da equipe, que sejam criados colegiados nos hospitais e que estes profissionais possam participar das tomadas de decisões já que estas afetam diretamente o desempenho da sua função. (PIMENTA, 2012)

É necessário articular os interesses dos profissionais e dos usuários, levando em consideração as particularidades de cada região de saúde para que a experiência dos usuários seja cada vez mais exitosa e o mesmo obtenha mais sucesso em seu tratamento. Porém, os estudos explicitam que existem diversos desafios e dificuldades a serem superadas nesse processo de crescimento e aperfeiçoamento do sistema único de saúde, no que diz respeito à gestão.(VIANNA, 2009; PIMENTA,2012; BARRETO, 2012; TRAD, 2009; GUIZARDI, 2009; MAGALHAES, 2004).

A falta de diálogo entre as várias escalas de poder dentro das instituições, é um problema na implantação da gestão participativa apontado pelos artigos encontrados, pois os atores envolvidos não participaram previamente das discussões e não estão familiarizados com as características desse modelo. (PIMENTA, 2012; BARRETO, 2012)

Dessa forma se faz necessário fomentar no grupo a importância que cada indivíduo tem para a instituição, enfatizando suas qualidades e proporcionando a superação de seus defeitos em união com a equipe; capacitar os profissionais para atuarem na gestão dos serviços, dando exclusividade à contratação de pessoas graduadas em gestão hospitalar ou com especialização em gestão dos serviços de saúde; proporcionar um ambiente digno aos funcionários seja ele gestor ou das equipes de atendimento direto ao paciente são importantes estratégias para valorizar a gestão participativa. (BERNARDES, 2012)

\section{CONCLUSÃO}

A partir da revisão elaborada foi possível explorar e conhecer de forma mais consistente a gestão participativa e como essa se desenvolve no Brasil, a qual é muito mais complexa do que o simples ato de delegar funções ou escutar sobre os anseios, desejos e receios dos profissionais de uma determinada instituição ou instância governamental. Ela consiste em uma re-estruturação de todo o modo de gerir a que estar-se habituado. 
Os resultados desse estudo apontam para a importância da gestão participativa, e do empenho dos trabalhadores. Revelaram, também, que há dificuldades, mas os desafios podem ser superados e que experiências exitosas existem em várias cidades, pois a Gestão Participativa mostra uma alternativa viável para resolução de conflitos no SUS, além de possibilidade de envolver todos os atores para participação de idéias e sugestões.

Destaca-se a dificuldade de encontrar estudos sobre esta temática, diante disso aponta-se a necessidade de desenvolvimento de pesquisas, além de mais relatos de experiência. Há a necessidade, também, de maior número de investigação por parte dos gestores. Assim, sugere-se que novos estudos sejam realizados acerca da temática no âmbito da gestão.

\section{REFERÊNCIAS}

1. BRASIL a. Ministério da Saúde. Política Nacional de Gestão Estratégica e Participação no SUS. Brasília; Brasil. Ministério da Saúde; 2009. 41 p. (Série B. Textos básicos de saúde).

2. BRASIL b. Lei no 8080 , de 19 de setembro de 1990. Dispõe sobre as condições para a promoção, proteção e recuperação da saúde, a organização e o funcionamento dos serviços correspondentes e dá outras providências. Diário Oficial da União. Brasília, 20 set. 1990; Seção 1.

3. BRASIL c. Lei 8142 , de 28 de dezembro de 1990. Dispõe sobre a participação da comunidade na gestão do Sistema Único de Saúde (SUS) e sobre as transferências intergovernamentais de recursos financeiros na área da saúde e dá outras providências. Diário Oficial da União. Brasília, 28 dez. 1990.

4. CUNHA, P. F.; MAGAJEWSKI, F. Gestão participativa e valorização dos trabalhadores: avanços no âmbito do SUS. Saude soc., São Paulo , v. 21, supl. 1, May 2012. Disponível em<http://www.scielo.br/scielo.php?script=sci_arttext\&pid=S0104-

$12902012000500006 \&$ Ing=en\&nrm=iso>. Acesso em 01 Dez 2013.

5. BEZERRA, L. C. A.ET AL . Identificação e caracterização dos elementos constituintes de uma intervenção: pré-avaliação da política ParticipaSUS. Ciênc. saúde coletiva, Rio de Janeiro , v. 17, n. $\quad 2012$.

<http://www.scielo.br/scielo.php?script=sci_arttext\&pid=S1413-

$81232012000400011 \&$ lng=en\&nrm=iso>. Acesso em 30 nov 2013.

6. PIMENTA, APARECIDA LINHARES. A construção de colegiados de gestão: a experiência de gestão da Secretaria Municipal de Saúde analisada por um ator político implicado. Saude soc., São Paulo , v. 21, supl. 1, May $2012 \quad$. $\quad$ Disponível em <http://www.scielo.br/scielo.php?script=sci_arttext\&pid=S0104$12902012000500003 \&$ Ing=en\&nrm=iso>. Acesso em 30 nov 2013.

7. BERNARDES, ANDREA ET AL . Framing the difficulties resulting from implementing a Participatory Management Model in a public hospital. Rev. Latino-Am. Enfermagem, Ribeirão Preto , v. 20, n. 6, Dec. $2012 \quad$ Disponível em 
<http://www.scielo.br/scielo.php?script=sci_arttext\&pid=S0104-

11692012000600017\&Ing=en\&nrm=iso>. Acesso em 30 nov 2013.

8. BARRETO, IVANA CRISTINA DE HOLANDA CUNHA ET AL. Gestão participativa no SUS e a integração ensino, serviço e comunidade: a experiência da Liga de Saúde da Família, Fortaleza, CE. Saude soc., São Paulo , v. 21, supl. 1, May 2012 . Disponível em $<$ http://www.scielo.br/scielo.php?script=sci_arttext\&pid=S0104-

$12902012000500007 \&$ Ing=en\&nrm=iso>. Acesso em 30 nov 2013.

9. CARDOSO, CÍNTIA GARCIA; HENNINGTON, ÉLIDA AZEVEDO. Trabalho em equipe e reuniões multiprofissionais de saúde: uma construção à espera pelos sujeitos da mudança. Trab. educ. saúde (Online), Rio de Janeiro , v. 9, supl. 1, 2011 . Disponível em<http://www.scielo.br/scielo.php?script=sci_arttext\&pid=S1981-

$77462011000400005 \&$ Ing=en\&nrm=iso>. Acesso em 30 nov 2013.

10. TRAD, LENY ALVES BOMFIM; ESPERIDIAO, MONIQUE AZEVEDO. Gestão participativa e corresponsabilidade em saúde: limites e possibilidades no âmbito da Estratégia de Saúde da Família. Interface (Botucatu), Botucatu , v. 13, supl. 1, 2009 . Disponível em <http://www.scielo.br/scielo.php?script=sci_arttext\&pid=S141432832009000500008\&lng=en\&nr m=iso>. Acesso em 30 nov 2013.

11. VIANNA, MARIA LUCIA TEIXEIRA WERNECK; CAVALCANTI, MARIA DE LOURDES; CABRAL, MARTA DE PINA. Participação em saúde: do que estamos falando? . Sociologias, Porto Alegre, $\mathrm{n}$. 21, June 2009 . Disponível em <http://www.scielo.br/scielo.php?script=sci_arttext\&pid=S1517$45222009000100010 \&$ Ing=en\&nrm=iso>. Acesso em 30 nov 2013.

12. GUIZARDI, FRANCINI LUBE. Do controle social à gestão participativa: interrogações sobre a participação política no SUS. Trab. educ. saúde, Rio de Janeiro, v. 7, n. 1, June 2009 . Disponível em $<$ http://www.scielo.br/scielo.php?script=sci_arttext\&pid=S1981$77462009000100002 \&$ Ing=en\&nrm=iso>. Acesso em 30 nov 2013.

13.GOULART, BETHANIA FERREIRA; FREITAS, MARIA IMACULADA DE FÁTIMA. A implicação de trabalhadores de ambulatórios municipais, em Uberaba, Minas Gerais, Brasil, na reorganização de serviços preconizada pelo Sistema Único de Saúde. Cad. Saúde Pública, Rio de Janeiro , v. 24, n. 9, Sept. 2008 . Disponível em <http://www.scielo.br/scielo.php?script=sci_arttext\&pid=S0102311X2008000900018\&lng=en\&nrm=iso>. Acesso em 30 nov 2013.

14. PEDROSA, JOSÉ IVO DOS SANTOS. Educação popular em saúde e gestão participativa no SUS. Revista APS, São Paulo, v. 11, n.3, jul-set 2008.

15. MAGALHAES, ANA MARIA MULLER; DUARTE, ÊRICA ROSALBA MALLMANN. Tendências gerenciais que podem levar a enfermagem a percorrer novos caminhos. Rev. bras. enferm., $\begin{array}{lllll}\text { Brasília } \quad \text { v. } & \text { 47, n. Aug. } & 2004 . & \text { Disponível }\end{array}$ em<http://www.scielo.br/scielo.php?script=sci_arttext\&pid=S0034-

$71672004000400004 \&$ Ing=en\&nrm=iso>. Acesso em 30 nov 2013. 